\title{
Prognostic Implications of Claudin 4 and Rock 1 in Triple Negative Breast Cancer
}

\author{
Shimaa Ahmed ${ }^{1, ~ *, ~ O l a ~ A . ~ H a r b ~}{ }^{1}$, Nashwa Nawar ${ }^{2}$ \\ ${ }^{1}$ Department of Pathology, Faculty of Medicine, Zagazig University, Zagazig, Egypt \\ ${ }^{2}$ Department of Clinical Oncology and Nuclear Medicine, Faculty of Medicine, Zagazig University, Zagazig, Egypt
}

Email address:

shimaaarafa@yahoo.com (S. Ahmed)

*Corresponding author

\section{To cite this article:}

Shimaa Ahmed, Ola A. Harb, Nashwa Nawar. Prognostic Implications of Claudin 4 and Rock 1 in Triple Negative Breast Cancer. Journal of Cancer Treatment and Research. Vol. 5, No. 6, 2017, pp. 95-103. doi: 10.11648/j.jctr.20170506.13

Received: January 20, 2017; Accepted: February 22, 2017; Published: December 28, 2017

\begin{abstract}
Background: Triple-negative breast cancer (TNBC) is associated with characteristically poor prognosis. Tumor invasion and metastasis lead to high mortality of patients with breast cancer. The identification of biomarkers that allow early detection of metastasis is essential for therapeutic success in the treatment of breast cancer. This study aimed to asses the immune-histochemical expression of Rock-1 and claudin-4 in triple negative breast cancer and comparing them with classic prognostic parameters as age, grade, lymph node state, distant metastasis and stage to evaluate their prognostic significance in triple negative breast cancer. Material and methods: Forty cases of triple negative breast cancer were examined immunohistochemically using antibodies against claudin 4 and ROCK 1. Results: There was a difference in the expression of claudin 4 and Rock 1 among different clinicopathological parameters. A statistically significant relationship was found between high claudin 4 expression and higher age at time of diagnosis, advanced tumor stage, presence of distant metastasis and increased number of nodal involvement $(\mathrm{p}=0.041,0.006,0.001$ and $<0.001$ respectively). A highly statistically significant relationship was detected between high Rock 1 expression and increased number of nodal involvement $(\mathrm{p}<0.001)$. Also the expression of Rock 1 was different according to different age groups, tumor grades, tumor stages and presence or absence of distant metastasis but this difference was statistically insignificant ( $\mathrm{p}=0.388,0.602,0.699$ and 0.944 respectively). Conclusion: our data suggest that claudin 4 and ROCK 1 are biomarkers of poor prognosis of patients with triple negative breast cancer.
\end{abstract}

Keywords: Triple Negative Breast Cancer, Claudin 4, Rock 1, Immunohistochemistry, Prognosis

\section{Introduction}

Breast cancer is one of the most common malignant tumors in women, more than $1,300,000$ cases and 450,000 deaths each year worldwide [1].

Breast cancer rates are increasing in Egypt, due to aging of the population, increased age at the time of first pregnancy, decrease in number of children and in breast feeding, and a move toward high-calorie Western diets [2].

Management of breast cancer depends on clinical and pathologic features of the tumor, including patient age, tumor size, histologic type and grade, lymph node stage, and lympho-vascular invasion [3].

Molecular markers such as estrogen receptor (ER), progesterone receptor (PR), and human epidermal growth factor receptor 2 (HER2) have been proven to provide therapeutic, predictive, and prognostic value [4].

Triple-negative breast cancer (TNBC) is defined by the loss of expression of receptor ER, PR, and Her2neu expressions and associated with biological aggressive-eness and poor prognosis. It represents approximately $10 \%$ to $17 \%$ of all breast carcinomas [5].

It is of particular interest to clinicians and research-hers due to their characteristically poor prognosis and resistance to existing molecularly-targeted treatment modalities, such as endocrine therapy (e.g., tamoxifen and aromatase inhibitors) for hormone receptor-positive disease or trastuzumab for human epidermal growth factor receptor-2 (HER2)-positive 
disease, leaving cyto-toxic chemotherapy as the principal systemic treatment [6].

The metastases from TNBC tend to be more aggressive with common visceral, hepatic, central nervous system, and lung involvement [7]. The identification of biomarkers that allow early detection of metastasis is essential for therapeutic success in the treatment of breast cancer [8].

The Rho-associated serine-threonine protein kinase-1 (ROCK-1) is a major kinase effector protein of Rho GTP ase signaling, correlated to the processes of invasion, angiogenesis and tumor aggressiveness [9].

ROCK becomes activated when it selectively binds to the active GTP-bound form of Rho. The action of this Rho/ROCK signalling pathway has been shown to be associated with tumour progression by regulation of actin cytoskeletal reorganization and the formation of focal adhesion $[10,11]$.

ROCKs also have an important role in cell migration by enhancing cell contractility and are required for tail retraction of cancer cells [12].

Abnormal highly expression of Rock 1 gene is detected in a variety of tumor, such as prostate and breast cancer also it plays a role in tumor cell invasion and metastasis [13-15].

Claudins are major integral membrane proteins of tight junction proteins and include 23 family members have been identified in humans [1].

Altered expression of several claudin proteins, in particular claudin-4 has been detected in various cancers as ovarian carcinoma and bladder cancer [16-18].

\section{Materials and Methods}

A total of 40 cases of formalin-fixed, paraffin-embedded specimens of triple-negative primary invasive breast carcinoma were included in this study. The cases were obtained retrospectively, from the files of the Pathology. Department, Faculty of Medicine, and Zagazig University during the period from December 2010 to May 2014. All cases underwent modified radical mastectomies. None of the cases had received chemo-therapy or radiotherapy prior to surgery.

All clinical data including age, size, grade, nodal status and distant metastasis were obtained from patient's files. Also receptor status(ER, PR, and Her 2) were obtained from their pathology reports.

\section{I- Histopathologic examination}

Tumor specimens were fixed in $10 \%$ buffered formalin and embedded in paraffin. Consecutive $4 \mathrm{~mm}$ sections were prepared and stained with hematoxylin and eosin for histopathological examination.

All the slides were re-evaluated according to WHO classifications of breast and female genital system tumors [19]. Grading was done based on Nottingham system. Staging was performed according to the International Union against Cancer TNM Classification [20].

II-Immunohistochemical staining:

Formalin fixed paraffin-embedded specimens were serially cut into Sections of 3-5 mm thickness sections for IHC), mounted on positively charged slides, deparaffinized in xylene, and rehydrated in a descending grades of alcohol. Sections were boiled in citrate buffer ( $\mathrm{pH} \mathrm{6.0)}$ for $20 \mathrm{~min}$ and then washed in PBS ( $\mathrm{pH}$ 7.3). For antigen retrieval, slides were immersed in ready to use Dako target retrieval solution (ph 6.0), then boiled in this solution in a microwave for 20 minutes. Thereafter, blocking of endogenous peroxidase activity with $3 \%$ hydrogen peroxide for $10 \mathrm{~min}$ at room temperature was carried out. The slides were then incubated at 2-8 C with primary antibodies: claudin 4, Rabbit Polyclonal antibody at a dilution of 1:50 (Thermo Fisher Scientific Lab Vision Corporation, Fremont, CA 94538, USA); ROCK 1 Rabbit monoclonal antibody at a dilution of 1:50 (Thermo Fisher Scientific Lab Vision Corporation, Fremont, CA 94538, USA). Incubation with a secondary antibody and product visualization were performed (DakoCytomation) with diaminobenzidine substrate (Research Genetics, Huntsville, Alabama, USA) as the chromogen. The slides were finally counterstained with Mayer's hematoxylin (BioGenex Laboratories, San Ramon, California, USA) and washed once each with distilled water and PBS. Human tonsil specimens and human colon tissue were used as positive controls for claudin 4 and ROCK 1 antibodies, respectively. Negative controls, obtained by substitution of primary antibodies with blocking buffer, were included within each slide batch.

Assessment of Immunohistochemical staining results:

1- Evaluation of claudin 4 immunostaining:

Claudin 4 was cytoplasmic in distribution. Immunoreactivity was assessed in at least 5 high-power fields at $\times 400$ magnification and based on combined score of the intensity ( 0 , no stain; 1 , weak; 2 , moderate; and 3, strong) and the percentage of stained tumor cells $(0,<5 \% ; 1,5 \%-25 \% ; 2$, $26 \%-50 \%$; and $3,>51 \%$ ). The 2 scores were multiplied to give an overall score of 0 to 9 , of which 0 was considered negative; 1 to 2 was considered weak; 3 to 6 , moderate; and 9, strong staining. Negative and weak expression was considered as low claudin expression, whereas moderate and strong as high claudin expression [21].

So a cut-off inferior or equal to 2 was considered as negative [22-23].

\section{2- Evaluation of ROCK 1 immunostaining:}

ROCK 1 was expressed in the cytoplasm. "Positive expression" was defined as $>10 \%$ positively stained cells under $200 \times$ microscopic field; $\leq 10 \%$ was defined as "negative expression [24].

\section{Statistical Analysis}

Categorical variables were expressed as a number (percentage). Percent of categorical variables were compared using the Pearson's Chi-square $\left(\chi^{2}\right)$ test. Receiver Operating Characteristic (ROC) curves were obtained to calculate the optimized cutoff point for Claudin 4 and ROCK 1 scores to reach the best compromise in the prediction of poor prognosis. The cutoff point with maximum sensitivity and specificity (validity) is used as the recommended cutoff point and also Area under Curve (AUC) was calculated. All tests 
were two sided, $\mathrm{p}$-value $<0.05$ was considered significant. All statistics were performed using SPSS 22.0 for windows (SPSS Inc., Chicago, IL, USA).

The staining results were evaluated in a double-blinded manner by two independent pathologists.

\section{Results}

\section{I-Patients' characteristics}

AQ10 The study included forty patients with their age ranged from 43 to 73 years. Most patients $(82.5 \%)$ were $>50$ years. Most carcinomas were grade II $(45 \%)$ or grade III (42.5), with most cases (87.5\%) associated with positive nodal involvement. The clinicopathological data are shown in Table 1 Figure $(2,5)$.

II- Expression of claudin 4 in TNBC cases and its association with clinicopathological parameters:

High levels of claudin 4 cytoplasmic expression were detected in $62.5 \%$ of TNBC cases and the remaining cases $(37.5 \%)$ showed low levels of claudin 4 expression. There was a difference in the expression of claudin 4 among different clinicopathological parameters. A statistically significant relationship was found between high claudin 4 expression and higher age at time of diagnosis, advanced tumor stage, presence of distant metastasis and increased number of nodal involvement $(\mathrm{p}=0.041,0.006,0.001$ and $<$ 0.001 respectively) (Table 2) Figure $(4,5)$.

III-Expression of Rock 1 in TNBC cases and its

Association with clinicopathological parameters:

High expression of Rock 1 was detected in $57.5 \%$ of TNBC cases and low expression was detected in $42.5 \%$. There was a difference in the expression of Rock 1 among different clinicopathological parameters. A highly statistically significant relationship was detected between high Rock 1 expression and increased number of nodal involvement $(\mathrm{p}<0.001)$ Also the expression of Rock 1 was different according to different age groups, tumor grades, tumor stages and presence or absence of distant metastasis but this difference was statistically insignificant $(\mathrm{p}=0.388$, 0.602, 0.699 and 0. 944 respectively) (Table 3) Figure $(3,5)$.

IV: Diagnostic performance of Claudin 4 and ROCK 1 scores as a predictor for poor prognosis of triple negative breast cancer patients:

The sensitivity of claudin 4 and ROCK 1 in predicting the prognosis was $80 \%$ and $65.7 \%$ respectively. While the sensitivity of claudin 4 and ROCK 1 together was $82.8 \%$. The specificity was $100 \%$ for both claudin 4 and ROCK 1 when done either separately or in combination (Table 4) Figure (3, 4, and 5). Claudin 4 x ROCK 1

Table 1. Clinicopathological features of studied cases $(N=40)$.

\begin{tabular}{|c|c|c|c|c|c|}
\hline & No. & (\%) & & No. & $(\%)$ \\
\hline \multicolumn{2}{|l|}{ Age (years) } & \multicolumn{4}{|c|}{ Lymph node (N) } \\
\hline$\leq 50$ year & 7 & $17.5 \%$ & No & 5 & $12.5 \%$ \\
\hline$>50$ year & 33 & $82.5 \%$ & N1 & 7 & $17.5 \%$ \\
\hline \multicolumn{2}{|l|}{ Tumor grade } & N2 & 15 & $37.5 \%$ & \\
\hline GI & 5 & $12.5 \%$ & N3 & 13 & $32.5 \%$ \\
\hline GII & 18 & $45 \%$ & & & \\
\hline GIII & 17 & $42.5 \%$ & & & \\
\hline \multicolumn{3}{|c|}{ Tumor stage $(\mathrm{T})$} & \multicolumn{3}{|c|}{ Distant Metastasis (M) } \\
\hline $\mathrm{T} 1(<2 \mathrm{~cm})$ & 5 & $12.5 \%$ & M0 & 28 & $70 \%$ \\
\hline $\mathrm{T} 2(2-5 \mathrm{~cm})$ & 20 & $50 \%$ & M1 & 12 & $30 \%$ \\
\hline $\mathrm{T} 3(>5 \mathrm{~cm})$ & 15 & $37.5 \%$ & & & \\
\hline
\end{tabular}

Categorical variables were expressed as a number (percentage).

Table 2. Relationship between proportion of tumor cells expressing Claudin 4 and different clinicopathological parameters of patients (N=40).

\begin{tabular}{|c|c|c|c|c|c|c|c|}
\hline & \multirow{3}{*}{ Total } & \multicolumn{4}{|c|}{ Claudin expression } & \multirow{3}{*}{$\chi^{2}$} & \multirow{3}{*}{ p-value } \\
\hline & & \multicolumn{2}{|c|}{ Low expression $(\leq 2)(n=15)$} & \multicolumn{2}{|c|}{ High expression $(>2)(n=25)$} & & \\
\hline & & No. & $\%$ & No. & $\%$ & & \\
\hline \multicolumn{8}{|l|}{ Age (years) } \\
\hline$\leq 50$ year & 7 & 5 & $71.4 \%$ & 2 & $28.6 \%$ & \multirow{2}{*}{4.167} & \multirow{2}{*}{0.041} \\
\hline$>50$ year & 33 & 10 & $30.3 \%$ & 23 & $69.7 \%$ & & \\
\hline \multicolumn{8}{|l|}{ Tumor grade } \\
\hline GI & 5 & 5 & $100 \%$ & 0 & $0 \%$ & \multirow{3}{*}{21.037} & \multirow{3}{*}{$<0.001$} \\
\hline GII & 18 & 10 & $55.6 \%$ & 8 & $44.4 \%$ & & \\
\hline GIII & 17 & 0 & $0 \%$ & 17 & $100 \%$ & & \\
\hline \multicolumn{8}{|c|}{ Tumor stage (T) } \\
\hline $\mathrm{T} 1(<2 \mathrm{~cm})$ & 5 & 5 & $100 \%$ & 0 & $0 \%$ & \multirow{3}{*}{10.347} & \multirow{3}{*}{0.006} \\
\hline $\mathrm{T} 2(2-5 \mathrm{~cm})$ & 20 & 7 & $35 \%$ & 13 & $65 \%$ & & \\
\hline $\mathrm{T} 3(>5 \mathrm{~cm})$ & 15 & 3 & $20 \%$ & 12 & $80 \%$ & & \\
\hline \multicolumn{8}{|c|}{ Lymph node $(\mathrm{N})$} \\
\hline No & 5 & 5 & $100 \%$ & 0 & $0 \%$ & 23.827 & $<0.001$ \\
\hline
\end{tabular}




\begin{tabular}{|c|c|c|c|c|c|c|c|}
\hline & \multirow{3}{*}{ Total } & \multicolumn{4}{|c|}{ Claudin expression } & \multirow{3}{*}{$\chi^{2}$} & \multirow{3}{*}{ p-value } \\
\hline & & \multicolumn{2}{|c|}{ Low expression $(\leq 2)(n=15)$} & \multicolumn{2}{|c|}{ High expression $(>2)(n=25)$} & & \\
\hline & & No. & $\%$ & No. & $\%$ & & \\
\hline $\mathrm{N} 1$ & 7 & 6 & $85.7 \%$ & 1 & $14.3 \%$ & & \\
\hline $\mathrm{N} 2$ & 15 & 4 & $26.7 \%$ & 11 & $73.3 \%$ & & \\
\hline N3 & 13 & 0 & $0 \%$ & 13 & $100 \%$ & & \\
\hline \multicolumn{8}{|c|}{ Distant Metastasis (M) } \\
\hline M0 & 28 & 15 & $53.6 \%$ & 13 & $46.4 \%$ & \multirow{2}{*}{10.286} & \multirow{2}{*}{0.001} \\
\hline M1 & 12 & 0 & $0 \%$ & 12 & $100 \%$ & & \\
\hline
\end{tabular}

Qualitative data are presented as number (\%); $\chi 2$ Chi-square test; $\mathrm{p}<0.05$ is significant.

Table 3. Relationship between proportion of tumor cells expressing ROCK 1 and different clinicopathological parameters of patients $(N=40)$.

\begin{tabular}{|c|c|c|c|c|c|c|c|}
\hline & \multirow{3}{*}{ Total } & \multicolumn{4}{|c|}{ ROCK 1 expression } & \multirow{3}{*}{$\chi^{2}$} & \multirow{3}{*}{ p-value } \\
\hline & & \multicolumn{2}{|c|}{ Low expression $(\leq 10 \%)(n=17)$} & \multicolumn{2}{|c|}{ High expression $(>10 \%)(n=23)$} & & \\
\hline & & No. & $\%$ & No. & $\%$ & & \\
\hline \multicolumn{8}{|l|}{ Age (years) } \\
\hline$\leq 50$ year & 7 & 4 & $57.1 \%$ & 3 & $42.9 \%$ & \multirow{2}{*}{0.744} & \multirow{2}{*}{0.388} \\
\hline$>50$ year & 33 & 13 & $39.4 \%$ & 20 & $60.6 \%$ & & \\
\hline \multicolumn{8}{|l|}{ Tumor grade } \\
\hline GI & 5 & 3 & $60 \%$ & 2 & $40 \%$ & \multirow{3}{*}{1.016} & \multirow{3}{*}{0.602} \\
\hline GII & 18 & 8 & $44.4 \%$ & 10 & $55.6 \%$ & & \\
\hline GIII & 17 & 6 & $35.3 \%$ & 11 & $64.7 \%$ & & \\
\hline \multicolumn{8}{|c|}{ Tumor stage $(\mathrm{T})$} \\
\hline $\mathrm{T} 1(<2 \mathrm{~cm})$ & 5 & 3 & $60 \%$ & 2 & $40 \%$ & \multirow{3}{*}{0.716} & \multirow{3}{*}{0.699} \\
\hline $\mathrm{T} 2(2-5 \mathrm{~cm})$ & 20 & 8 & $40 \%$ & 12 & $60 \%$ & & \\
\hline $\mathrm{T} 3(>5 \mathrm{~cm})$ & 15 & 6 & $40 \%$ & 9 & $60 \%$ & & \\
\hline \multicolumn{8}{|c|}{ Lymph node (N) } \\
\hline No & 5 & 5 & $100 \%$ & 0 & $0 \%$ & \multirow{4}{*}{19.075} & \multirow{4}{*}{$<0.001$} \\
\hline N1 & 7 & 6 & $85.7 \%$ & 1 & $14.3 \%$ & & \\
\hline N2 & 15 & 5 & $33.3 \%$ & 10 & $66.7 \%$ & & \\
\hline N3 & 13 & 1 & $7.7 \%$ & 12 & $92.3 \%$ & & \\
\hline \multicolumn{8}{|c|}{ Distant Metastasis (M) } \\
\hline M0 & 28 & 12 & $42.9 \%$ & 16 & $57.1 \%$ & \multirow{2}{*}{0.005} & \multirow{2}{*}{0.944} \\
\hline M1 & 12 & 5 & $41.7 \%$ & 7 & $58.3 \%$ & & \\
\hline
\end{tabular}

Qualitative data are presented as number (\%); $\chi^{2}$ Chi-square test; $p<0.05$ is significant.

Table 4. Diagnostic performance of Claudin 4 and ROCK 1 scores as a predictor for poor prognosis of triple negative breast cancer patients; ROC curve Analysis.

\begin{tabular}{|c|c|c|c|c|c|c|c|}
\hline IHC & $\begin{array}{l}\text { Cut-off } \\
\text { values }\end{array}$ & $\begin{array}{l}\text { SN\% } \\
(95 \% \text { CI) }\end{array}$ & $\begin{array}{l}\text { SP\% } \\
(95 \% \mathrm{CI})\end{array}$ & $\begin{array}{l}\text { PPV\% } \\
(95 \% \text { CI) }\end{array}$ & $\begin{array}{l}\text { NPV\% } \\
(95 \% \text { CI) }\end{array}$ & $\begin{array}{l}\text { Accuracy } \\
(95 \% \text { CI) }\end{array}$ & $\begin{array}{l}\text { AUC } \\
(95 \% \mathrm{CI})\end{array}$ \\
\hline Claudin 4 & $>1.5$ & $\begin{array}{l}80 \% \\
(63.1-91.6 \%)\end{array}$ & $\begin{array}{l}100 \% \\
(47.8-100 \%)\end{array}$ & $\begin{array}{l}100 \% \\
(87.7-100 \%)\end{array}$ & $\begin{array}{l}41.7 \% \\
(14.2-73.6 \%)\end{array}$ & $\begin{array}{l}82.5 \% \\
(68.2-96.8 \%)\end{array}$ & $\begin{array}{l}0.934 \ddagger \\
(0.809-0.988)\end{array}$ \\
\hline ROCK 1 & $>9$ & $\begin{array}{l}65.7 \% \\
(47.8-80.9 \%)\end{array}$ & $\begin{array}{l}100 \% \\
(47.8-100 \%)\end{array}$ & $\begin{array}{l}100 \% \\
(85.2-100 \%)\end{array}$ & $\begin{array}{l}29.4 \% \\
(10.3-56 \%)\end{array}$ & $\begin{array}{l}70 \% \\
(52.7-87.3 \%)\end{array}$ & $\begin{array}{l}0.820 \S \\
(0.666-0.923)\end{array}$ \\
\hline $\begin{array}{l}\text { Claudin } 4+ \\
\text { ROCK } 1\end{array}$ & $>10$ & $\begin{array}{l}82.8 \% \\
(66.4-93.4 \%)\end{array}$ & $\begin{array}{l}100 \% \\
(47.8-100 \%)\end{array}$ & $\begin{array}{l}100 \% \\
(88.1-100 \%)\end{array}$ & $\begin{array}{l}45.5 \% \\
(15.6-78 \%)\end{array}$ & $\begin{array}{l}85 \% \\
(71.5-98.4 \%)\end{array}$ & $\begin{array}{l}0.903 * \\
(0.767-0.974)\end{array}$ \\
\hline $\begin{array}{l}\text { Claudin } 4 x \\
\text { ROCK } 1\end{array}$ & $>11.2$ & $\begin{array}{l}88.6 \% \\
(73.3-96.8 \%)\end{array}$ & $\begin{array}{l}100 \%) \\
(47.8-100 \%)\end{array}$ & $\begin{array}{l}100 \% \\
(88.1-100 \%)\end{array}$ & $\begin{array}{l}55.6 \% \\
(21.2-86.3 \%)\end{array}$ & $\begin{array}{l}90 \% \\
(78.7-100 \%)\end{array}$ & $\begin{array}{l}0.949 \bullet \\
(0.829-0.993)\end{array}$ \\
\hline
\end{tabular}

$\ddagger \mathrm{p}<0.001 ; \S \mathrm{p}<0.001 ; * \mathrm{p}<0.001 ; \cdot \mathrm{p}<0.001$.

ROC curve: Receiver Operating Characteristic curve;

SN: Sensitivity;

SP: Specificity;

PPV: Positive Predictive Value;

NPV: Negative Predictive Value;

AUC: Area under Curve; $95 \%$

CI: $95 \%$

Confidence Interval; $p<0.05$ is significant. Figure (1) 


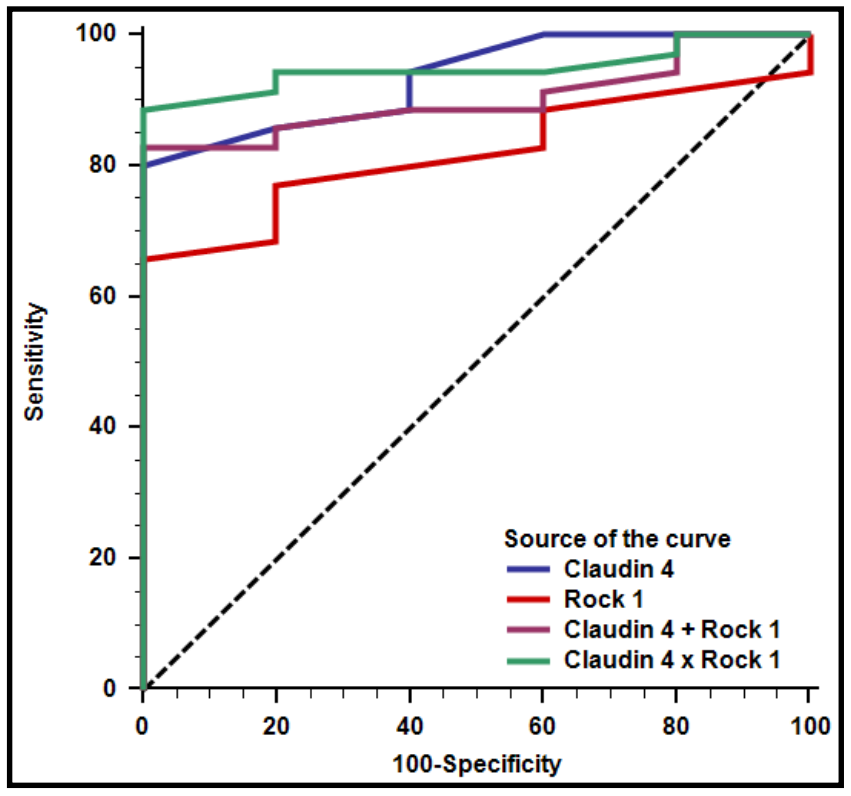

Figure 1. Receiver Operating Characteristic (ROC) curves of Claudin 4 and ROCK 1 scores as a predictor for poor prognosis of triple negative breast cancer patients.

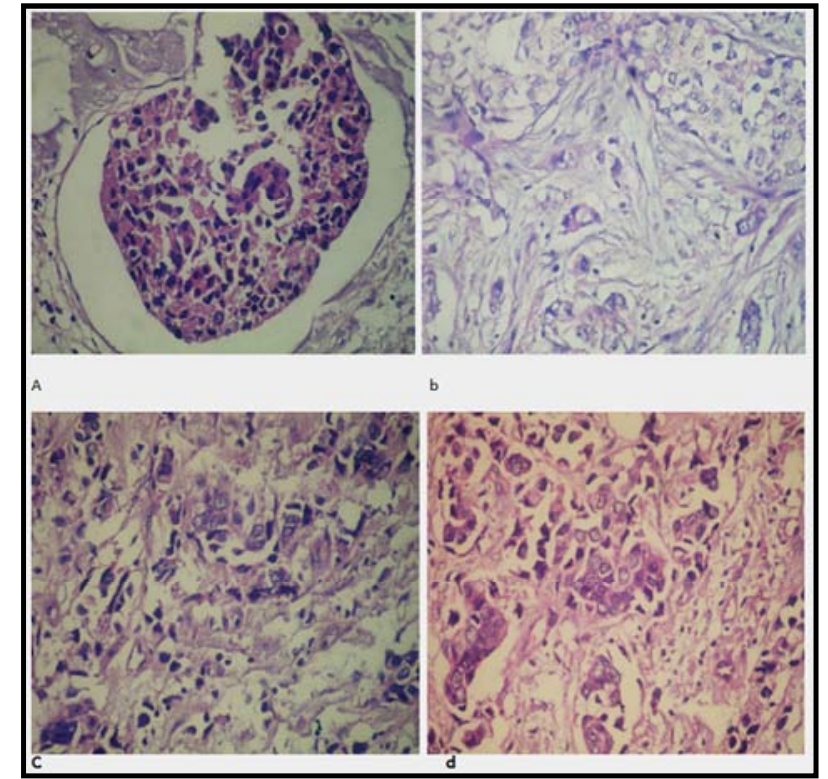

Figure 2. (Hematoxylin \& eosin; $x 400$ ) (a) Carcinoma in situ showing large proliferating pleomorphic malignant cells (b) Grade I infiltrating duct carcinoma, not otherwise specified showing solid trabeculae of malignant cells with little pleomorphism and low mitotic activity (c) Grade II infiltrating duct carcinoma showing large pleomorphic malignant cells (d) Grade III infiltrating duct carcinoma showing highly pleomorphic malignant cells with frequent mitosis.

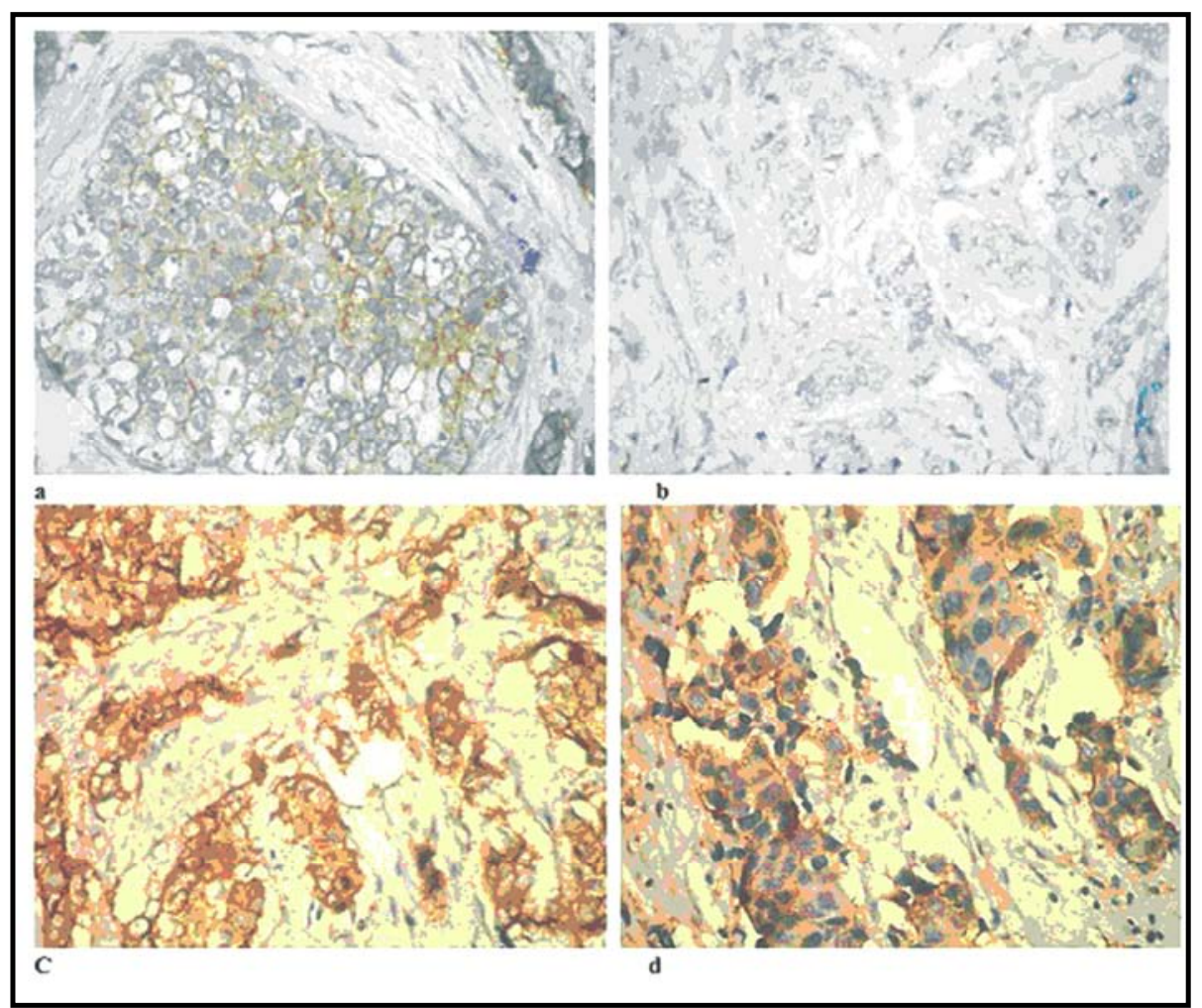

Figure 3. (Rock 1; x400) (a) Carcinoma in situ showing high levels of Rock 1 cytoplasmic expression (b)Grade I infiltrating duct carcinoma showing low levels of Rock 1 cytoplasmic expression (c)Grade II infiltrating duct carcinoma showing high levels of Rock 1 cytoplasmic expression. (d) Grade III infiltrating duct carcinoma showing high levels of Rock 1 cytoplasmic expression. 


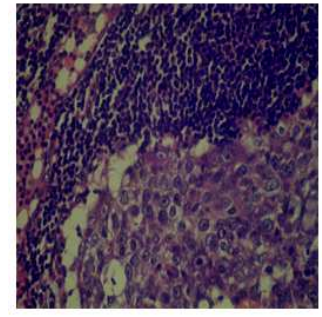

a

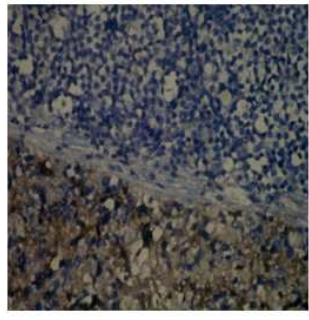

b

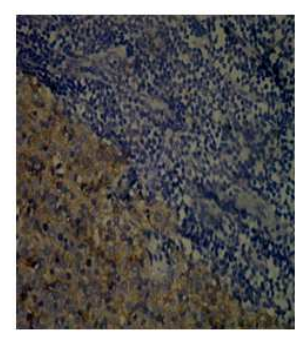

$\mathrm{c}$

Figure 4. (Claudin 4; 400 )(a) Carcinoma in situ showing high levels of Claudin cytoplasmin expression (b) Grade I infiltrating duct carcinoma showing low levels of Claudin 4 cytoplasmic expression (c) Grade II infiltrating duct carcinoma showing high levels of Claudin 4 cytoplasmic expression (d) Grade III infiltrating duct carcinoma showing high levels of Claudin 4 cytoplasmic expression.

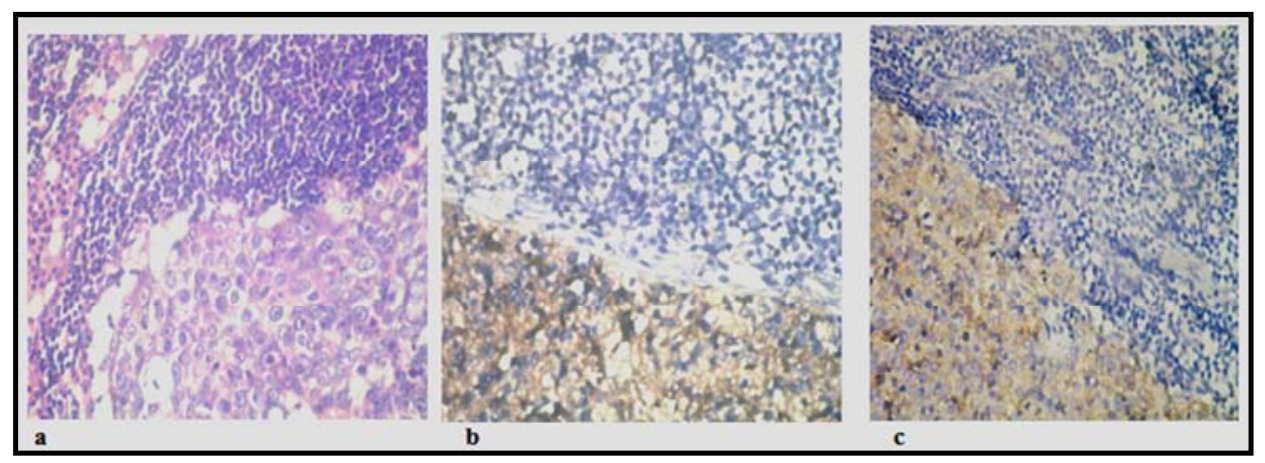

Figure 5. Lymph node showing metastasis from infiltrating duct carcinoma of the breast (a) (hematoxylin \&eosin x400) (b) (Rock 1; $x 400)$ showing high levels of Rock 1 cytoplasmic expression (c) (Claudin 4; 400$)$ showing high levels of Claudin 4 cytoplasmic.

\section{Discussion}

Breast cancer is the most common cancer among women [25]. Tumor invasion and metastasis affect more than $90 \%$ of patients with breast cancer and are the main factors that contribute to high mortality [26]. These processes occur due to the weak cytoskeletal structure allowing the low anchorage of neoplastic cells [27].

Tight junctions consist of transmembrane proteins, such as claudins (CLDNs), occludin, and many peripheral membrane proteins; CLDNs play crucial roles in the formation and maintenance of the tight junctions [28]. They are connected with the actin cytoskeleton and participate in intracellular signaling [29]. In this context, down regulation or up regulation of CLDNs might have a role in cancer development [30]. Alterations of CLDNs have been noted in several tumors such as colorectal, ovarian and breast cancer $[31,32]$.

The up-regulation of claudin- 4 has also been associated with tumorigenesis, and their expression is up-regulated in several malignancies, including breast, gastric, pancreatic, prostate and uterine cancers [33].

In the current study, High levels of claudin 4 cytoplasmic expression were detected in $62.5 \%$ of TNBC cases and this expression was significantly associated higher age at time of diagnosis, higher tumor grade, advanced tumor stage, increased number of nodal involvement and presence of distant metastasis $(\mathrm{p}<0.05)$

Our results are consistent with those of Mona and Marwa study who found that $66.1 \%$ of TNBCs express high levels of claudin 4; and this expression was significantly associated with large tumor size, high histologic grade, nodal involvement, and distant metastasis. Furthermore, they found that increased cytoplasmic localization of overexpressed claudin 4 was observed in the studied TNBC positive cases. This may be attributed to that claudin 4 is not functioning correctly in which the tight junctions were disorganized, and paracellular Permeability was increased [34].

Similarly Blanchard and Kulka [33, 35] studied claudin 4 expressions in TNBC tumors and found association between the expression of claudin 4 and high-grade TNBC tumors. These findings go with other previous studies that found association of the expression of claudin 4 with high-grade breast cancer [36, 37]. Also Lanigan described the association of increased expression of claudin 4 with high tumor grade, ER-negative tumors, and poor prognosis [38].

Our results were different from those reported by Garbar and Kolokytha [39-40] who found that claudin 4 could be a biomarker of favorable prognosis in TNBC and negative claudin 4 TNBC show a poor breast cancer-specific survival curves.

Rho-associated coiled-coil containing protein kinase (ROCK) belongs to a family of serine/threonine kinases and is one of the best characterized downstream effectors of Rho GTP ases [41]. Via ROCK activation, Rho GTP ases have been implicated in multiple cellular processes including motility, morphogenesis, polarity, cell division, and cell adhesion [42]. In humans, there are two known ROCK isoforms-ROCK 1 and ROCK 2. ROCK over-expression has been implicated in the progression of many tumor types, 
including bladder carcinoma, hepatocellular carcinoma, and breast carcinoma [43, 44, 45] the increased expression of ROCK-1 is related to the presence of tumor metastasis and its inhibition is a novel approach for treating breast cancer [46].

In the current study, High levels of Rock 1 were detected in $57.5 \%$ of TNBC cases. There was a difference in the expression of Rock 1 among different clinico-pathological parameters. A highly statistically significant relationship was detected between high Rock 1 expression and increased number of nodal involvement $(p<0.001)$. Also the expression of Rock 1 was different according to different age groups, tumor grades, tumor stages and presence or absence of distant metastasis but this difference was statistically insignificant.

Our results are consistent with Bottinoa who found higher expression of ROCK 1 in women with lymph node involvement compared to those without lymph node involvement $(p=0.007)$. Also they found variation in the expression of ROCK-1 between different clinical tumor stages, but without a statistically significant difference ( $p>$ 0.05 ). In addition, they found strong labeling areas of ROCK1 protein in the group of women who had metastases but without a statistically significant difference [47].

Similarly Liu assessed the expression of ROCK 1 in human breast cancer specimens and cell lines and found high expression of ROCK-1 in metastatic human mammary tumors (from patients with nodal metastasis) compared with non-metastatic tumors (node-negative). Also they reported that ROCK 1 was markedly elevated in the tumorigenic and metastatic cell lines, compared with the tumorigenic but nonmetastatic cell lines. These observations suggest that overexpression of ROCK 1 may contribute to the metastatic features of breast cancer cells. They also claimed that the inhibition of ROCK-1 can suppress the growth, migration and metastasis of these cells [46].

The same result was shown in a study by Patel, in which they studied the effect of RKI-1447 in Human breast and lung cancer cell lines and found that RKI-1447 was highly selective at inhibiting ROCK-mediated cytoskeleton reorganization, migration, invasion and anchorage-independent tumor growth of breast cancer cells. Also, RKI-1447 inhibited tumor growth and caused tumor regression in animal models with little side effects [48].

Lane reported that high levels of ROCK I is correlated significantly with shorter overall survival; mean survival 103.20 months (74.37-132.03 months 95\% CI) vs. 139.87 months (130.31-149.43 months 95\% CI) for those with low levels of ROCK I; $p=0.0304$. They further tested the effects of a ROCK inhibitor (Y27632) on the cells and found a significant decrease in invasiveness when compared with those untreated cells $(78.6 \pm 6.42$ vs. $131.6 \pm 20.8 \mathrm{p}<0.005)$. From their findings they suggested that ROCK I is a potential therapeutic target in human breast cancer, with the possible use of ROCK inhibitors acting as antimetastatic chemotherapeutic agents [49].

A study by Croft also reported increased expression of ROCK-1 in human cancers with invasive and metastatic phenotypes and suggested that ROCK inhibitors would be useful antimetastatic and antiangiogenic chemotherapeutic agents in tumors associated with elevated ROCK I expression [50].

\section{Conclusion}

Claudin 4 and ROCK 1 can be considered as a potential marker for unfavorable prognosis of patients with triple negative breast cancer and can predict clinical outcome of triple negative breast cancer patients. Also they can be targeted for future therapy. However claudin 4 is more sensitive than ROCK 1 in predicting the prognosis. It is better to do them together because of higher sensitivity.

\section{Abbreviations}

TNBC: Triple-negative breast cancer; ER: estrogen receptor; PR: progesterone receptor; HER 2: human epidermal growth factor receptor 2; CLDNs: Claudins; ROCK: Rho-associated coiled-coil containing protein kinase.

\section{References}

[1] Madhu Lal-Nag and Patrice J Morin; The claudins. Genome Biol. 2009, 10, 235. Published online 2009 Aug 26. doi:10.1186/gb-2009-10-8-23.

[2] Lanigan F, McKiernan E, Brennan DJ, Hegarty S, Millikan RC, McBryan J, Jirstrom K, Landberg G, Martin F, Duffy MJ. Increased claudin-4 expression is associated with poor prognosis and high tumour grade in breast cancer Int $\mathrm{J}$ Cancer. 2009 May 1; 124(9).

[3] Goldhirsch E. P, Winer A. S., Coates, R. D. Gelber, M. Piccart-Gebhart, B. Thürlimann, H.-J. Senn, Panel members, Kathy S. Albain, et al. (2013) Personalizing the treatment of women with early breast cancer: highlights of the St Gallen International Expert Consensus on the Primary Therapy of Early Breast Ann Oncol.; 24(9):2206-2223.

[4] Won JR, Gao D, Chow C, et al. (2013) A survey of immunohistochemical biomarkers for basal-like breast cancer against a gene expression profile gold standard. Mod Pathol.; 26(11):1438-1450.

[5] Kutomi, G. T. Ohmura, Y. Suzuki, (2012) "Clinicopathological characteristics of basal type breast cancer in triple-negative breast cancer," Journal of Cancer Therapy, vol. 3 , pp. 836-840.

[6] Györffy B, Lanczky A, Eklund AC, Denkert C, Budczies J, Li Q, et al. (2010). An online survival analysis tool to rapidly assess the effect of 22,277 genes on breast cancer prognosis using microarray data of 1,809 patients. Breast Cancer Res Treat.; 123:725-731.

[7] Foulkes WD, Smith IE, Reis-Filho JS. (2010)Triple-negative breast cancer. N Engl J Med.; 363:1938-1948.

[8] Fan J, Deng X, Gallagher JW, Huang H, Huang Y, Wen J, et al. (2012). Monitoring the progression of metastatic breast cancer on nanoporous silica chips. Philos Trans A: Math Phys Eng Sci; 370:2433-47. 
[9] Schackmann RC, Van Amersfoort M, Haarhuis JH, Vlug EJ, Halim VA, Roodhart JM, et al. (2011). Cytosolic p120-catenin regulates growth of metastatic lobular carcinoma through Rock 1-mediated anoikis resistance. J Clin Invest; 121:317688.

[10] Singh A. B., Sharma A., Dhawan P. (2010). Claudin family of proteins and cancer: An overview. J. Oncol.; 2010:541957.

[11] Ricardo S, Gerhard R, Cameselle-Teijeiro JF, Schmitt F, Paredes J. Claudin (2012). Expression in breast cancer: high or low, what to expect? Histol Histopathol. Oct; 27(10):128395 .

[12] Fielding CJ, Fielding PE: (2000). Cholesterol and caveolae: structural and funct ional relationships. Biochim Biophys Acta, 1529:210-22.

[13] Guan R, Xu X, Chen M, Hu H, Ge H, Wen S, Zhou S, Pi R: (2013). Advances in the studies of roles of Rho/Rho-kinase in diseases and the development of its inhibitors. Eur $J$ Med Chem, 70:613-622.

[14] Lock FE, Ryan KR, Poulter NS, Parsons M, Hotchin NA: (2012). Differential regulation of adhesion complex turnover by ROCK 1 and ROCK 2. PLoS One, 7(2): e31423.

[15] Liu X, Choy E, Hornicek FJ et al. (2012). ROCK 1 as a potential therapeutic target in osteosarcoma. J Orthop Res 2011; 29:1259-66.

[16] Singh, A. B.; Sharma, A.; Dhawan, P. (2010). Claudin family of proteins and cancer: An overview. J. Oncol., 541957.

[17] Zhu, Y.; Brannstrom, M.; Janson, P. O et al. (2006). Differences in expression patterns of the tight junction proteins, claudin $1,3,4$ and 5 , in human ovarian surface epithelium as compared to epithelia in inclusion cysts and epithelial ovarian tumours. Int. J Cancer, 118, 1884-1891.

[18] Boireau, S.; Buchert, M.; Samuel, M. S.; Pannequin, J.; Ryan, J. L.; Choquet, A.; Avances, C.; Ernst, M.; et al. (2007). DNAmethylation-dependent alterations of claudin- 4 expression in human bladder carcinoma. Carcinogenesis, 28, 246-258.

[19] Tavassoli FA, Devilee P.; (2003). World Health Organization Classification of Tumors: Pathology and Genetics of Tumors of the Breast and Female Genital Organs. Lyon, France: IARC Press.

[20] Greene FL, Pgae DL, Felming D, et al. (2002). American Joint Comettee on Cancer (AJCC) cancer staging manual. 6th ed. New York: Springer-Verlag.

[21] Sheehan G M, Kallakury BV, Sheehan CE, Fisher HA, Kaufman Jr RP, Ross JS. (2007). Loss of claudins- 1 and -7 and expression of claudins- 3 and -4 correlate with prognostic variables in prostatic adenocarcinomas. Hum Pathol; 38:564 9.

[22] Perou CM. (2010). Molecular stratification of triple-negative breast cancers. Oncologist.; 16:39-48.

[23] Strehl J, Wachter D, Fasching P, et al. (2011). Invasive breast cancer: recognition of molecular subtypes. Breast Care; 6(4):256-264

[24] Zhang J, He X, Ma Y, Liu Y, Shi H, Guo W, Liu L. (2015). Overexpression of ROCK 1 and ROCK 2 inhibits human laryngeal squamous cell carcinoma. Int J Clin Exp Pathol; $8(1): 244-251$.
[25] International Agency for Research Cancer (IARC). (2012). http://globocan.iarc.fr/facysheets/cancers/ breast.asp [accessed 03.19.12].

[26] Fagan-Solis KD, Schneider SS, Pentecost BT, Bentley BA, Otis CN, Gierthy JF, et al. (2012). The ras homolog gene family, member A (Rho A) pathway mediates MMP-2 and MMP-9-independent invasive behavior in a triple-negative breast cancer cell line. J Cell Biochem; 114:1385-94.

[27] Ortíz-López L, Morales-Mulia S, Ramírez-Rodríguez G, Benítez- King G. (2009). ROCK-regulated cytoskeletal dynamics participate in the inhibitory effect of melatonin on cancer cell migration. J Pineal Res; 46:15-21.

[28] Tsukita S, Furuse M and Itoh M: (2001). Multifunctional strands in tight Junctions. Nat Rev Mol Cell Biol 2: 285-293.

[29] Ivanov AI, Nusrat A and Parkos CA: (2004) Endocytosis of epithelial apical junctional proteins by a clathrin-mediated pathway into a unique storage compartment. Mol Biol Cell 15: 176-188.

[30] Kominsky SL, Argani P, Korz D, Evron E, Raman V, Garrett E, Rein A, Sauter G, Kallioniemi OP and Sukumar S:(2003). Loss of the tight junction protein claudin-7 correlates with histological grade in both ductal carcinoma in situ and invasive ductal carcinoma of the breast. Oncogene 22: 20212033.

[31] Sawada N, Murata M, Kikuchi K, Osanai M, Tobioka H, Kojima T and Chiba H: (2003). Tight junctions and human diseases. Med Electron Microsc 36: 147-156.

[32] Hirohashi S and Kanai Y: (2003). Cell adhesion system and human cancer morphogenesis. Cancer Sci 94: 575-581.

[33] Kulka J, Szasz AM, Nemeth Z, et al. (2009) Expression of tight junction protein claudin-4 in basal-like breast carcinomas. Pathol Oncol Res; 15:59-64 [PMID: 18752049 .

[34] Mona A. Abd-Elazeem, Marwa A. Abd-Elazeem, (2015). Claudin 4 expression in triple-negative breast cancer: correlation with androgen receptors and Ki-67 expression Annals of Diagnostic Pathology Volume 19, Issue 1, Pages $37-42$.

[35] Blanchard AA, Skliris GP, Watson PH, et al. (2009). Claudins1,3 , and 4 protein expression in ER negative breast cancer correlates with markers of the basal phenotype. Virchows Arch; 454:647-56.

[36] Toke's AM, Kulka J, Paku S, et al. (2005). Claudin-1, -3 and -4 proteins and mRNA expression in benign and malignant breast lesions: a research study. Breast Cancer Res; 7: R 296305 .

[37] Ricardo S, Gerhard R, Cameselle-Teijeiro JF, et al. (2012). Claudin expression in breast cancer: high or low, what to expect? Histol Histopathol; 27(10):1283-95.

[38] Lanigan F, McKiernan E, Brennan DJ, et al. (2009). Increased claudin-4 expression is associated with poor prognosis and high tumor grade in breast cancer. Int J Cancer; 124:2088-97.

[39] Garbar C, Dudez O, Mombelli S, et al. (2014). Claudin-4 Immunohistochemical Expression Is an Independent Prognosis Factor in Triple-negative Breast Cancers. J Multidiscip Pathol.; 1(1):1-10. 
[40] Kolokytha P, Yiannou P, Keramopoulos D, et al. (2014). Claudin-3 and claudin-4: distinct prognostic significance in triple-negative and luminal breast cancer. Appl Immunohistochem Mol Morphol; 22(2):125-31.

[41] Narumiya S, Tanji M and Ishizaki T. (2009) Rho signaling, ROCK and mDia, in transformation, metastasis and invasion. Cancer Metastasis Rev; 28: 65-76.

[42] Jaffe AB and Hall A. Rho GTPases: (2005). Biochemistry and biology. Annu Rev Cell Dev Biol; 21: 247-269.

[43] Kamai T, Tsujii T, Arai K, Takagi K, Asami H, Ito Y and Oshima H. (2003). Significant association of Rho/ROCK pathway with invasion and metastasis of bladder cancer. Clin Cancer Res; 9: 2632-2641.

[44] Wong CC, Wong CM, Tung EK, Man K and Ng IO. (2009). Rho-kinase 2 is frequently overexpressed in hepatocellular carcinoma and involved in tumor invasion. Hepatology; 49: 1583-1594.

[45] Lane J, Martin TA, Watkins G, Mansel RE and Jiang WG. (2008). the expression and prognostic value of ROCK I and ROCK II and their role in human breast cancer. Int J Oncol; 33: 585-593.
[46] Liu S, Goldstein RH, Scepansky EM, Rosenblatt M. (2009). Inhibition of rho- associated kinase signaling prevents breast cancer metastasis to human bone. Cancer Res; 69:8742-51.

[47] Bottinoa J, Gelaleti GB, Maschioa LB Jardim-Perassia B V, Zuccaria DA. (2014). Immuno expression of ROCK-1 and MMP-9 as prognostic markers in breast cancer. Acta Histochemica 116 1367-1373.

[48] Patel RA, Forinash KD, Pireddu R, Sun Y, Sun N, Martin MP, et al. (2012). RKI- 1447 is a potent inhibitor of the Rhoassociated ROCK kinases with anti-invasive and antitumor activities in breast cancer. Cancer Res; 72:5025-34.

[49] Lane J, Martin TA, Watkins G, Mansel RE, Jiang WG. (2008). The expression and prognostic value of ROCK I and ROCK II and their role in human breast cancer. Int J Oncol; 33:585-93.

[50] Croft DR, Sahai E, Mavria G, Li S, Tsai J, Lee WM, et al. (2004). Conditional ROCK activation in vivo induces tumor cell dissemination and angiogenesis. Cancer Res; 64:89949001. 